Biochemistry

of Sulfur 


\title{
BIOCHEMISTRY OF THE ELEMENTS
}

\author{
Series Editor: Earl Frieden \\ Florida State University \\ Tallahassee, Florida
}

\begin{abstract}
Volume 1 BIOCHEMISTRY OF NONHEME IRON Anatoly Bezkorovainy

Volume 2 BIOCHEMISTRY OF SELENIUM

Raymond J. Shamberger

Volume 3 BIOCHEMISTRY OF THE ESSENTIAL

ULTRATRACE ELEMENTS

Edited by Earl Frieden

Volume 4 BIOCHEMISTRY OF DIOXYGEN

Lloyd L. Ingraham and Damon L. Meyer

Volume 5 PHYSICAL METHODS FOR INORGANIC BIOCHEMISTRY

John R. Wright, Wayne A. Hendrickson, Shigemasa Osaki, and Gordon T. James

Volume 6 BIOCHEMISTRY OF SULFUR

Ryan J. Huxtable
\end{abstract}

A Continuation Order Plan is available for this series. A continuation order will bring delivery of each new volume immediately upon publication. Volumes are billed only upon actual shipment. For further information please contact the publisher. 


\section{Biochemistry of Sulfur}

Ryan J. Huxtable

University of Arizona Health Sciences Center

Tucson, Arizona

With a contribution by

W. Mark Lafranconi

The Procter and Gamble Company

Miami Valley Laboratories

Cincinnati, Ohio

\section{Springer Science+Business Media, LLC}




\section{Library of Congress Cataloging in Publication Data}

Huxtable, Ryan.

Biochemistry of sulfur.

(Biochemistry of the elements; v. 6)

1. Sulphur-Physiological effect. 2. Sulphur-Metabolism. 3. Organosulphur compounds-Physiological effect. 4. Organosulphur compounds-Metabolism. I. Lafranconi, W. Mark. II. Title. III. Series.

\section{(c) Springer Science+Business Media New York 1986}

Originally published by Plenum Press, New York in 1986

Softcover reprint of the hardcover 1st edition 1986

Original figures $(1986$ Ryan J. Huxtable

\section{All rights reserved}

No part of this book may be reproduced, stored in a retrieval system, or transmitted in any form or by any means, electronic, mechanical, photocopying, microfilming, recording, or otherwise, without written permission from the Publisher 


\section{Preface}

There can be few elements with a biochemistry as coherent as that of sulfur. This important element is crucial to myriad aspects of metabolism, catalysis, and structure. The plurality of functions in which sulfur is involved derives squarely from the numerous oxidation states in which it may exist, some having great stability, some being capable of ready redox interconversions, and yet others having great instability. As a result, the flux of sulfur from the geosphere through the various kingdoms of life leaves few biochemical processes unaffected.

Although there are large gaps in the fabric of our basic knowledge of sulfur biochemistry, it is sufficiently framed to allow a unified and organized story, a story which many of the best-known names in biochemistry have helped to write. It has been both a task and a privilege to try and summarize this story, one that is enormous, complex, fastmoving, still developing and, above all, exciting. I suppose that no monographer of such a vast subject could be satisfied with his efforts. It is unfortunately probable that in attempting this task I have made as many errors as a Stilton cheese has blue streaks, and as many omissions as a Swiss cheese has holes. Perfection is not to be achieved in a monograph. Inasmuch as I have succeeded, the credit belongs to those whose efforts gave us the knowledge we have. Where I have failed, the fault is only mine.

All enzyme control numbers are taken from E. C. Webb, Enzyme Nomenclature (Academic Press, 1984).

Ryan J. Huxtable 


\section{Contents}

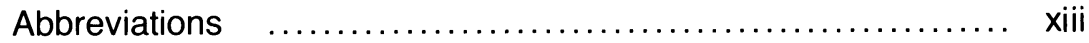

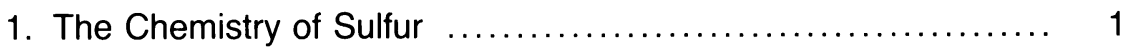

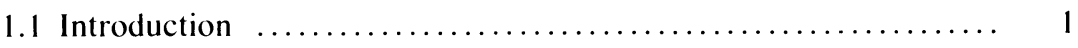

1.2 Characteristics of Sulfur Chemistry $\ldots \ldots \ldots \ldots \ldots \ldots \ldots \ldots \ldots, 3$

1.3 The Oxidation States of Sulfur $\ldots \ldots \ldots \ldots \ldots \ldots \ldots \ldots \ldots \ldots \ldots \ldots \ldots, 4$

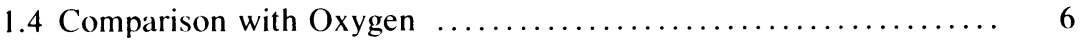

2. The Reduction of Sulfate and the Oxidation of Sulfide $\ldots \ldots \ldots \ldots 11$

2.1 Introduction $\ldots \ldots \ldots \ldots \ldots \ldots \ldots \ldots \ldots \ldots \ldots \ldots \ldots \ldots \ldots \ldots \ldots, 11$

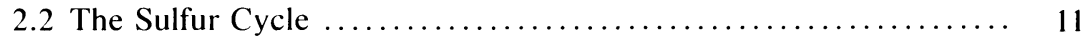

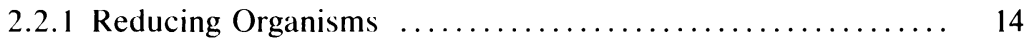

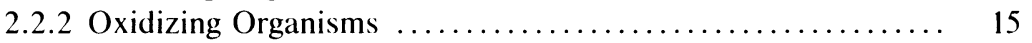

2.3 The Sulfuretum .................................... 16

2.4 Reduction of Sulfate to Inorganic Sulfide $\ldots \ldots \ldots \ldots \ldots \ldots \ldots \ldots$

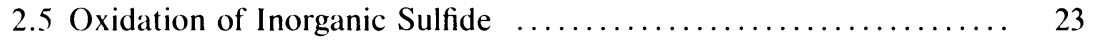

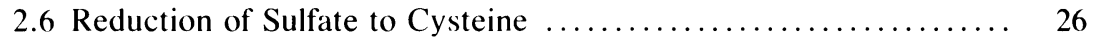

2.6.1 Transport of Sulfate ............................. 27

2.6.2 Activation of Sulfate ......................... 28

2.6.3 Assimilatory Reduction of Sulfate ................. 30

2.6.4 Carbon-Sulfur Bond Formation from Organic Intermediates 32

2.6.5 Carbon-Sulfur Bond Formation from Inorganic

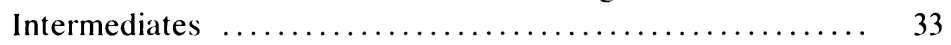

2.6.6 Assimilation by Dissimilatory Sulfate Reducers .......... 34

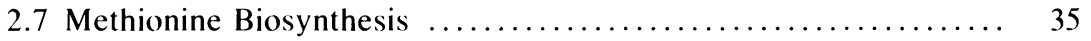

2.7.1 Methionine Biosynthesis in Enteric Bacteria $\ldots \ldots \ldots \ldots \ldots . .36$

2.7.2 Methionine Biosynthesis in Fungi .................. 41

2.7.3 Homocysteine Biosynthesis: Comparative Aspects ........ 44

2.7.4 Regulation of Methionine Biosynthesis ............... 45 
3. The Metabolism and Functions of Methionine $\ldots \ldots \ldots \ldots \ldots \ldots, 63$

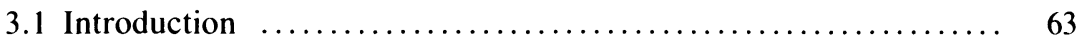

3.2 The Methionine-Homocysteine Cycle $\ldots \ldots \ldots \ldots \ldots \ldots \ldots \ldots .63$

3.2.1 Polyglutamate Regulation of the Cycle $\ldots \ldots \ldots \ldots \ldots \ldots \ldots .67$

3.2.2 The Methyl Trap Hypothesis ..................... 71

3.2.3 Regulation of Folate Metabolism by the Cycle ........... 74

3.2.4 Methyl Neogenesis and Methyl Salvage ................ 74

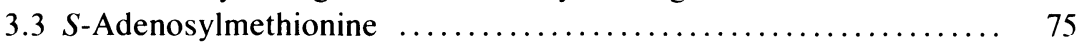

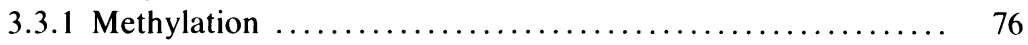

3.3.2 Polyamine Biosynthesis $\ldots \ldots \ldots \ldots \ldots \ldots \ldots \ldots \ldots \ldots \ldots$

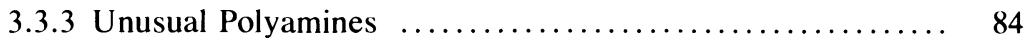

3.3.4 Metabolism of $S$-Adenosylmethionine $\ldots \ldots \ldots \ldots \ldots \ldots \ldots . \quad 85$

3.3.5 Metabolism of Methylthioadenosine .................. 88

3.4 Transsulfuration in Mammals $\ldots \ldots \ldots \ldots \ldots \ldots \ldots \ldots \ldots \ldots . \ldots \ldots$

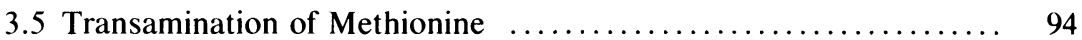

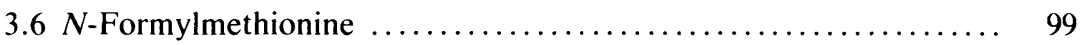

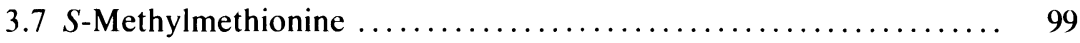

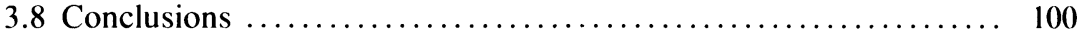

4. Taurine and the Oxidative Metabolism of Cysteine $\ldots \ldots \ldots \ldots \ldots 121$

4.1 Introduction $\ldots \ldots \ldots \ldots \ldots \ldots \ldots \ldots \ldots \ldots \ldots \ldots \ldots \ldots \ldots \ldots \ldots \ldots, 121$

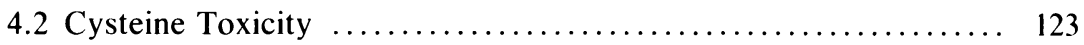

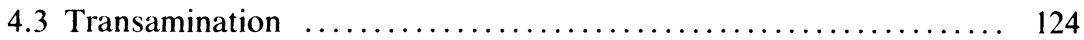

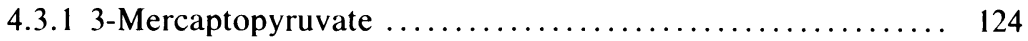

4.3.2 Sulfane Metabolism ............................ 124

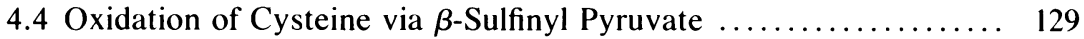

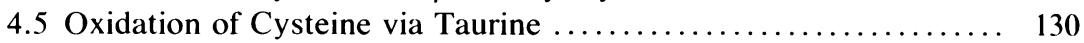

4.5.1 The Cysteine Sulfinic Acid Decarboxylase Route ........ 131

4.5.2 The Cysteic Acid Decarboxylase Route ............... 137

4.5.3 The Cysteamine Dioxygenase Route ................ 137

4.5.4 The PAPS Transferase Route ..................... 140

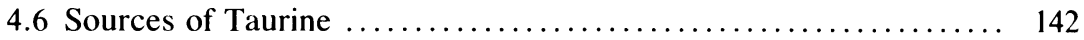

4.6.1 Levels .................................... 142

4.6.2 Diet versus Biosynthesis ....................... 142

4.6.3 Taurine-Conditionally Essential for Humans? .......... 146

4.7 Functions and Pharmacology of Taurine ................. 150

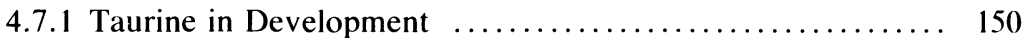

4.7.2 The Brain .................................... 152

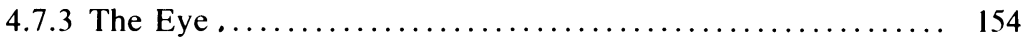

4.7.4 The Cardiovascular System ....................... 155

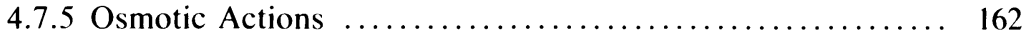




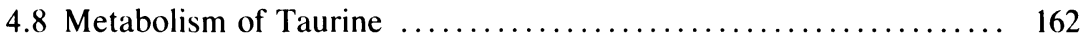

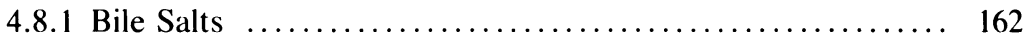

4.8 .2 Isethionic Acid ................................ 167

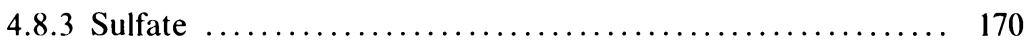

4.8.4 Taurine-Containing Peptides $\ldots \ldots \ldots \ldots \ldots \ldots \ldots \ldots \ldots \ldots \ldots$

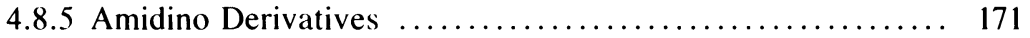

4.8.6 Xenobiotic Conjugates ......................... 172

4.8.7 Other Taurine Metabolites ....................... 173

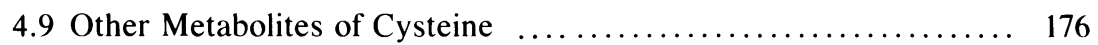

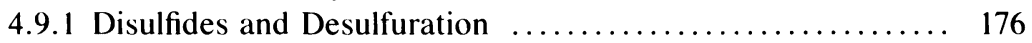

4.9.2 Cysteine Pigments ........................... 179

5. Thiols, Disulfides, and Thioesters ....................... 199

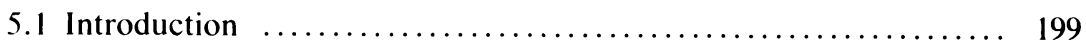

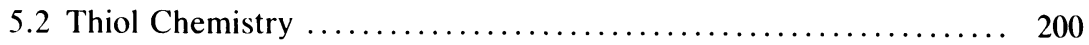

5.2.1 Hydrogen Bonding and Acidity ................... 201

5.2 .2 Metal Chelation ................................. 202

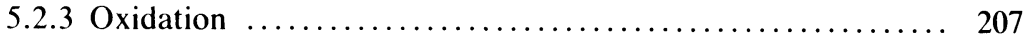

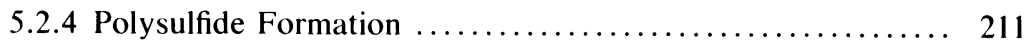

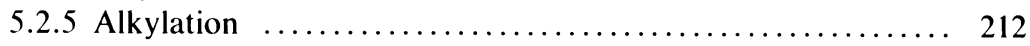

5.2.6 Hemimercaptals and Thiazolidines $\ldots \ldots \ldots \ldots \ldots \ldots \ldots . \ldots 213$

5.2.7 Other Addition Reactions ......................... 214

5.2 .8 Charge Transfer ................................. 215

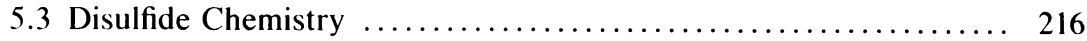

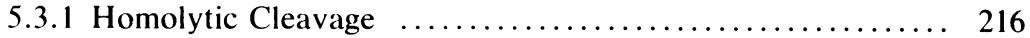

5.3 .2 Heterolytic Cleavage $\ldots \ldots \ldots \ldots \ldots \ldots \ldots \ldots \ldots \ldots \ldots . \ldots . \ldots \ldots$

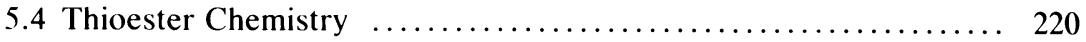

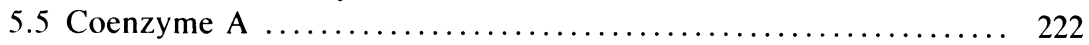

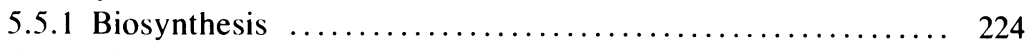

5.5.2 Dissimilation of Coenzyme A .................... 227

5.5.3 Formation of Coenzyme A Esters .................. 229

5.5.4 The Functions of Coenzyme A .................... 230

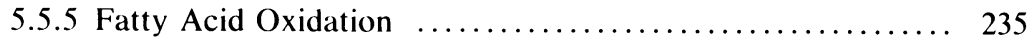

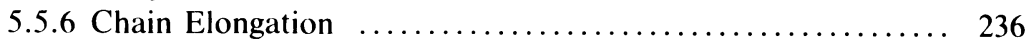

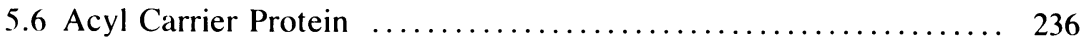

5.7 Other Phosphopantetheine-dependent Reactions $\ldots \ldots \ldots \ldots \ldots . . \ldots 244$

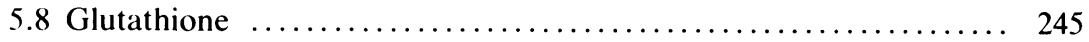

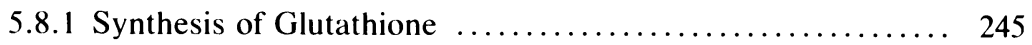

5.8.2 The Kinetics of Glutathione ....................... 246

5.8.3 The $\gamma$-Glutamyl Cycle $\ldots \ldots \ldots \ldots \ldots \ldots \ldots \ldots \ldots \ldots \ldots . \ldots \ldots \ldots$

5.8 .4 The Redox State ............................... 250

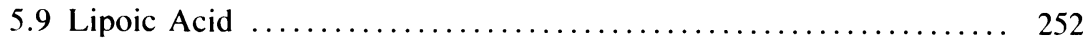




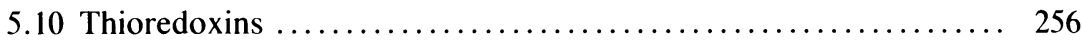

5.11 Dithiol-flavin Enzymes .......................... 257

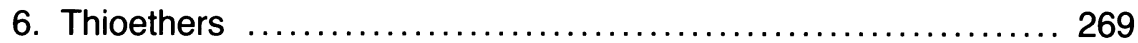

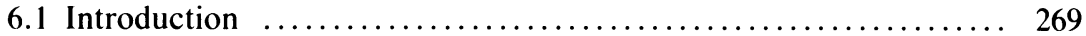

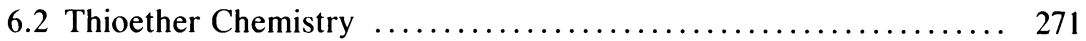

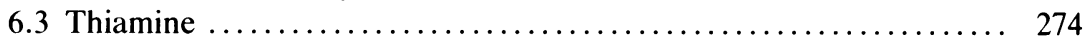

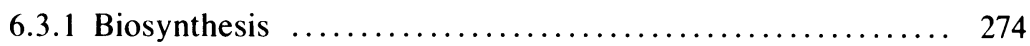

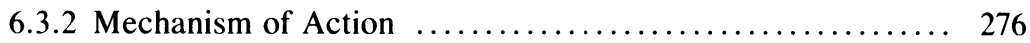

6.3.3 Thiamine Deficiency ........................... 279

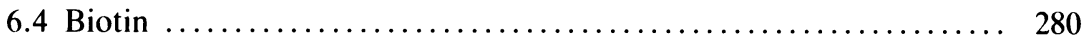

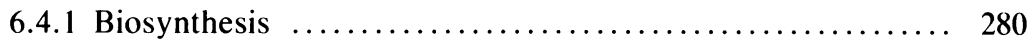

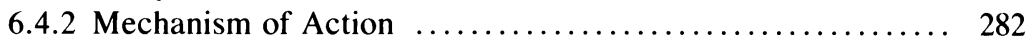

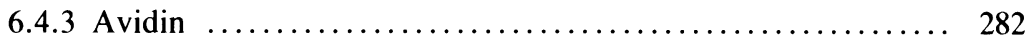

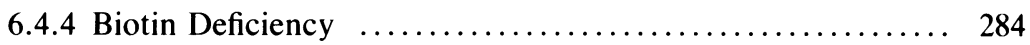

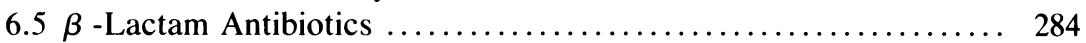

6.5.1 Biosynthesis ................................. 286

6.5.2 Mechanism of Action $\ldots \ldots \ldots \ldots \ldots \ldots \ldots \ldots \ldots \ldots \ldots \ldots$

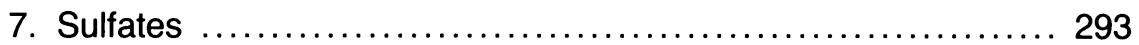

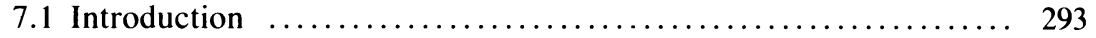

7.2 Sulfate Activation .................................... 297

7.2.1 Biosynthesis of PAPS ........................... 298

7.2 .2 Sulfate Availability ........................... 299

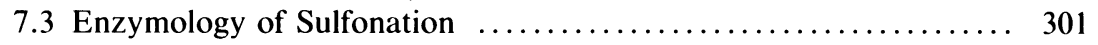

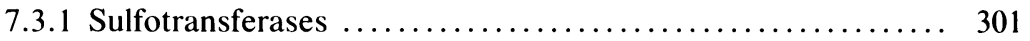

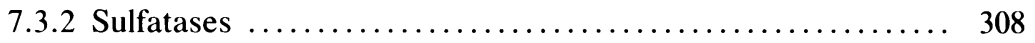

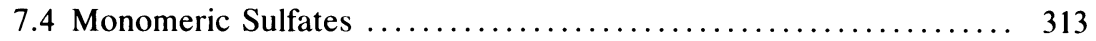

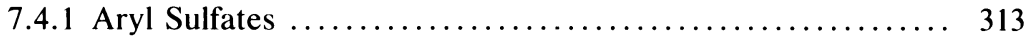

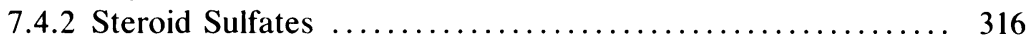

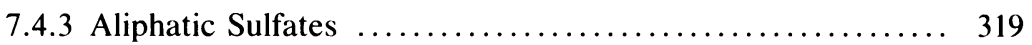

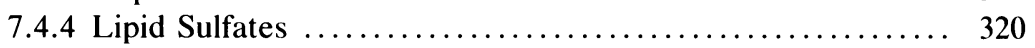

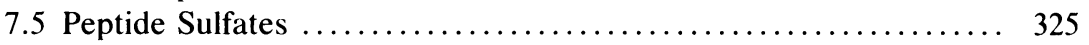

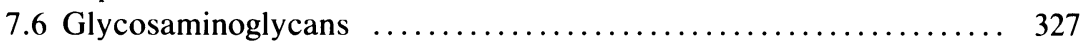

7.6.1 The Structures and Functions of the Glycosaminoglycans ... 330

7.6.2 Glycosaminoglycans and Intercellular Space ............ 333

7.6.3 The Chondroitins ............................. 337

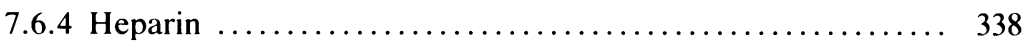

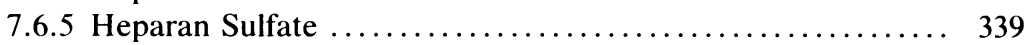

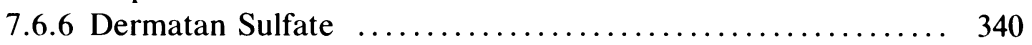

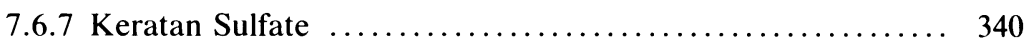




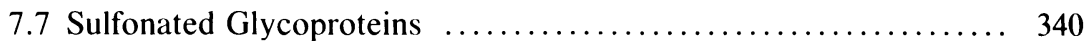

7.8 Algal Polysaccharide Sulfates $\ldots \ldots \ldots \ldots \ldots \ldots \ldots \ldots \ldots \ldots \ldots . \ldots \ldots 1$

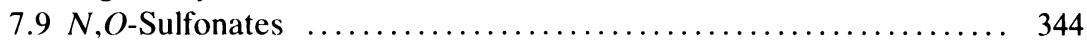

7.9.1 Hydroxamic Acid Sulfates $\ldots \ldots \ldots \ldots \ldots \ldots \ldots \ldots \ldots \ldots \ldots \ldots \ldots$

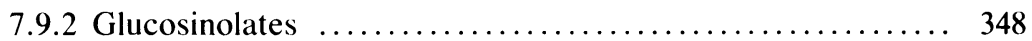

8. Inherited Disorders of Sulfur Metabolism .................. 359

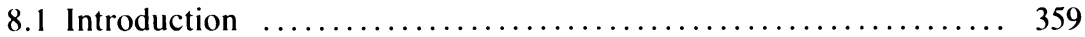

8.2 Disorders of Transsulfuration and the Met-Hcy Cycle ........ 360

8.2.1 5,10-Methylene Tetrahydrofolate Reductase Deficiency .... 364

8.2.2 Methyltetrahydrofolate Methyltransferase Deficiency ...... 365

8.2.3 Methionine Adenosyltransferase Deficiency ............ 366

8.2.4 Cystathionine $\beta$-Synthase Deficiency ............... 366

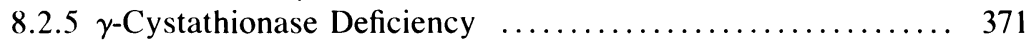

8.2.6 Sulfite Oxidase Deficiency ........................ 374

8.3 Disorders in GSH Synthesis and Regulation ............... 374

8.3.1 $\gamma$-Glutamylcysteine Synthase Deficiency $\ldots \ldots \ldots \ldots \ldots \ldots . \ldots 374$

8.3.2 GSH Synthase Deficiency ...................... 374

8.3.3 $\gamma$-Glutamyl Transpeptidase Deficiency $\ldots \ldots \ldots \ldots \ldots \ldots . \ldots 377$

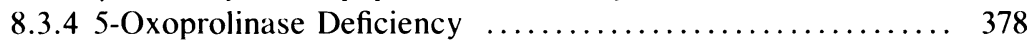

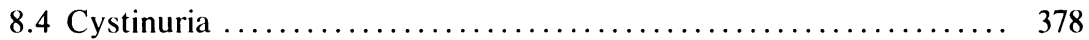

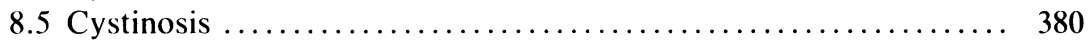

8.6 Lipid Sulfatase Deficiencies ......................... 381

8.6.1 Steroid Sulfate Sulfatase Deficiency ................ 382

8.6.2 Cerebroside Sulfate Sulfatase Deficiency ............. 386

8.7 Disorders of Glycosaminoglycan Catabolism .............. 387

8.7.1 Iduronate Sulfate Sulfatase Deficiency ............... 393

8.7.2 N-Acetylgalactosamine 4-Sulfate Sulfatase Deficiency ...... 394

8.7.3 Defects in Heparan Sulfate Metabolism ................ 394

8.7.4 Defects in Keratan Sulfate Metabolism .................. 396

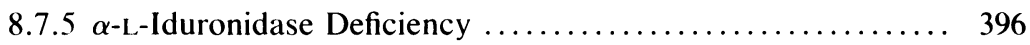

8.7.6 $\beta$-Glucuronidase Deficiency $\ldots \ldots \ldots \ldots \ldots \ldots \ldots \ldots \ldots \ldots \ldots \ldots$

8.8 Multiple Sulfatase Deficiency $\ldots \ldots \ldots \ldots \ldots \ldots \ldots \ldots \ldots \ldots \ldots . \ldots \ldots$

8.9 Undersulfation of Glycosaminoglycans . ................. 399

9. Sulfur and the Metabolism of Xenobiotics ..................4 413

Contributed by W. Mark Lafranconi

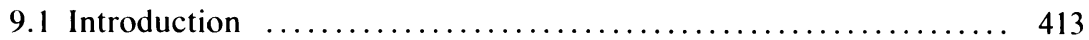

9.2 General Aspects of Xenobiotic Metabolism ................ 413

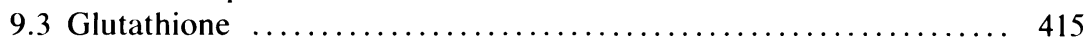

9.3.1 Chemistry .................................. 415 
9.3.2 Participation in Conjugation $\ldots \ldots \ldots \ldots \ldots \ldots \ldots \ldots \ldots \ldots, 417$

9.3.3 Scavenging of Free Radicals $\ldots \ldots \ldots \ldots \ldots \ldots \ldots \ldots \ldots, 422$

9.3.4 Metabolism of Formaldehyde ..................... 426

9.3.5 Metabolic Activation as a Result of GSH Conjugation ..... 427

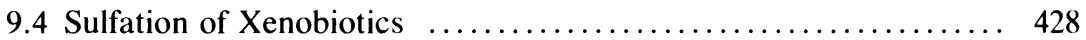

9.4.1 Enzymes Mediating Sulfate Conjugation $\ldots \ldots \ldots \ldots \ldots \ldots .430$

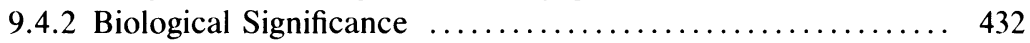

9.4.3 Metabolic Activation as a Result of Sulfate Conjugation ... 433

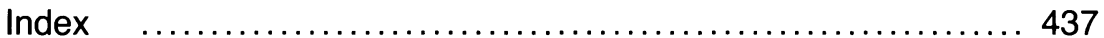




\section{Abbreviations}

\begin{tabular}{|c|c|}
\hline $\mathrm{ACP}$ & .. acyl carrier protein \\
\hline AdoHcy & .. $S$-adenosylhomocysteine \\
\hline AdoMet & $S$-adenosylmethionine \\
\hline APS $\ldots$ & ... adenosine phosphosulfate \\
\hline & , noiling point \\
\hline $\mathrm{CA}$ & (n) \\
\hline CNS $\ldots$ & $\ldots \ldots \ldots \ldots \ldots \ldots \ldots \ldots \ldots$ central nervous system \\
\hline CSA $\ldots$ & ... cysteine sulfinic acid \\
\hline Cys & .. cysteine \\
\hline Cysta & ... cystathionine \\
\hline DTT & .. dithiothreitol \\
\hline GSH & .. glutathione \\
\hline GSSG . & $\ldots \ldots \ldots \ldots$ oxidized glutathione \\
\hline h $\ldots . . .$. & nour \\
\hline Hcy & ..... homocysteine \\
\hline MeSAdo & .. methylthioadenosine \\
\hline Met $\ldots$. & .. methionine \\
\hline mol & $\ldots \ldots \ldots \ldots \ldots \ldots \ldots \ldots \ldots \ldots$ mole \\
\hline $\mathrm{mp}$ & $\ldots \ldots \ldots \ldots \ldots$ melting point \\
\hline MW & $\ldots \ldots \ldots \ldots \ldots \ldots \ldots$ molecular weight \\
\hline PAP & ........... phosphoadenosine phosphate \\
\hline PAPS & $\ldots \ldots \ldots \ldots$ phosphoadenosine phosphosulfate \\
\hline THF & 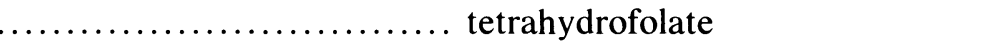 \\
\hline $\mathrm{SH}$ & .. sulfhydryl group \\
\hline
\end{tabular}

